\title{
Analysis and Evaluation of Influence of Vibration on Imaging of Aerial Camera
}

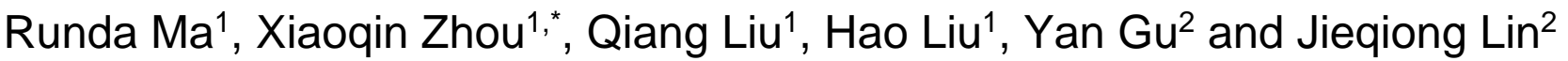 \\ ${ }^{1}$ School of Mechanical and Aerospace Engineering, Jilin University, China \\ ${ }^{2}$ School of mechanical Engineering, Changchun University of Technology, Changchun, Jilin \\ Province, China \\ ${ }^{*}$ Corresponding author
}

Keywords: Imaging Quality, Aerial Cameras, TDICCD, OTF, MTF, PTF, Vibration

\begin{abstract}
In order to quantitatively analyze the influence of vibration on the imaging quality of aerial cameras, the optical transfer function is used to evaluate the image of vibration on imaging quality. The dynamic Modulation Transfer Function(MTF) is used to evaluate the degree of blurring of the image. And the linear portion of the Phase Transfer Function(PTF) is used to characterize the geometric deformation of the image.
\end{abstract}

\section{Introduction}

Aerial photography is a long-range,non-contact target detection technology. Aerial camera as one of the main loads in the field of remote sensing. The aerial camera acquires ground scene information through optical imaging, which has the characteristics of strong timeliness, high measurement accuracy and wide detection area.[1,2]

Aviation remote sensing technology plays an increasingly important role in resource exploration, disaster monitoring and ground mapping[3]. When the aerial camera is photographed, the vibration of the aircraft components and the camera itself and the fluctuation of the vibration caused by airflow will affect the imaging quality of the camera and reduce the resolution of the image, especially for large-field and long-focus imaging systems[4].On the one hand, due to the large bandwidth and frequency-intensive vibration in the working environment, the vibration of the carrier will be transmitted to the optical system through the platform, causing the vibration of the optical system. On the other hand, because the system often uses mechanical rotation to achieve functions such as expanding the imaging range, assembly errors and transmission errors of mechanical components can cause vibration of the optical device[5,6].

This paper briefly introduces the root cause of image quality--image motion. And it is of great significance to analyze the influence of vibration on camera imaging quality and measurement accuracy for predicting the imaging and measurement performance of aeronautical cameras in the vibration environment and guiding the design of camera vibration isolation system.

\section{Image Motion}

At the moment of aerial photography exposure, the movement of the image on the imaging medium is called image motion. The image motion changes the shape and hue of the image, which reduces the sharpness of the image. When the image motion is large, the image is blurred.

The image motion in aerial camera photography is mainly caused by the movement of the aircraft with the aircraft at the moment of exposure[7]. The forward motion, unstable motion and selfvibration of the aircraft will produce image motion.

Taking the linear motion along the heading as an example, the aerial camera is affected by the flying speed of the aircraft when the ground scene is photographed, and the flight vibration of the aircraft is also affected, causing a relative displacement between the target object and the imaged medium. Causes the image to be blurred, called the image shift. As shown in Fig. 1, the aerial camera photographs the object B. During the exposure time $\Delta t$, the aircraft reaches point $\mathrm{A}^{\prime}$ from 
point $\mathrm{A}$, and the imaging on the $\mathrm{CCD}$ also changes from point $\mathrm{C}$ to point $\mathrm{C}^{\prime}$, and $\mathrm{CC}^{\prime}$ is image motion.

The pixels on the CCD sensor change depending on where the lens axis is swept. Set the image surface to be mounted at the focal length $f$. Define $\theta$ as the sweep angle of the optical axis. In the direction of flight, the image motion velocity due to the forward motion of the aircraft is

$$
V_{i}=V \frac{f}{H} \cos \theta
$$

where $V_{i}$ is the image motion velocity; $V$ is the flight velocity; $f$ is the focal length and $H$ is the flight height.

If the exposure time $\Delta t$ is determined, the magnitude of the image motion $\delta$ can be calculated.

$$
\delta=V \frac{f}{H} \cos \theta \Delta t
$$

\section{The Optical Transfer Function(OTF)}

In order to quantitatively analyze the influence of vibration on the imaging quality of aerial cameras, it is necessary to establish an evaluation model of the influence of vibration on the imaging quality of the camera. This paper uses OTF to establish this model.

The Optical Transfer Function(OTF) is a function that characterizes the relative change in modulation and lateral phase shift during imaging using spatial frequency as a variable. The optical transfer function is the filtering transformation of the spatial spectrum of the optical system.

The Optical Transfer Function(OTF) is an ideal method for evaluating the imaging quality of optical systems. It has a rigorous theory and can be extended to all aspects of imaging. For example, atmospheric disturbance, detector performance, vibration, etc. have an impact on the overall imaging performance of the system. According to the transfer function theory of the linear system, Robert D. Fiete[8,9] established the link model of the imaging system , evaluating the overall imaging performance of the system. It can be expressed in the following form:

$$
O T F_{\text {system }}=O T F_{\text {optic }} \times O T F_{\text {atmosphere }} \times O T F_{\mathrm{CCD}} \times O T F_{\text {vibration }} \times \cdots
$$

Where $O T F_{\text {system }}$ is the transfer function of the optical system, $O T F_{\text {atmosphere }}$ is the transfer function in the atmospheric transmission, $O T F_{C C D}$ is the transfer function of the CCD detector, and $O T F_{\text {vibration }}$ is the optical transfer function caused by the vibration.

\section{Evaluation Model of the Influence of Vibration on Imaging Quality of Aerial Cameras}

For an optical imaging system, a point on the object surface is modulated by the atmosphere, an optical system, a detector, etc., and a defocused spot having a certain width is formed on the image surface. The center of the defocused spot is the position of the object after geometric imaging. The defocused spot normalized energy distribution is the point spread function of the imaging system. As shown in Figure 1, when there is vibration image motion, the position of the image point changes. On the one hand, the width of the defocused spot becomes larger, making the image blurred, and on the other hand, the center position of the defocused spot changes, resulting in geometric distortion of the image. As shown in Figure 1. 


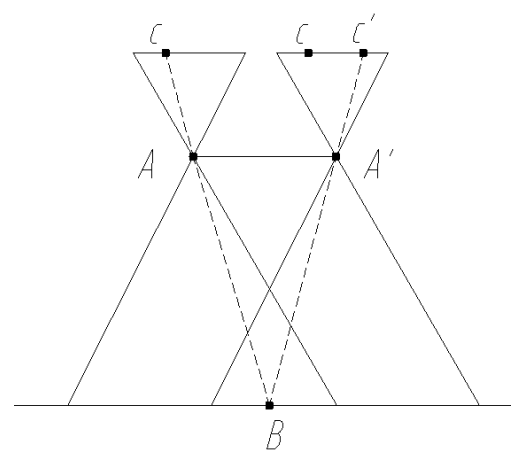

Figure 1. Image Motion Generation

For a one-dimensional space, the line spread function(LSF) is $h(X)$ when there is no vibration image motion. Let the vibration image motion to $x(t)$, at which point the diffusion function of the imaging system becomes $h(x)^{*} \delta[X-x(t)]$. The actual imaging result of the optical system is the superposition of energy during the exposure time. The line spread function should then be a normalized energy distribution[10]. It be expressed in the following form:

$$
g(X)=\frac{1}{t_{e}} \int_{t_{s}}^{t_{s}+t_{e}} h(X) * \delta[X-x(t)] d t
$$

where $t_{s}$ is the initial exposure time and $t_{e}$ is the exposure time.

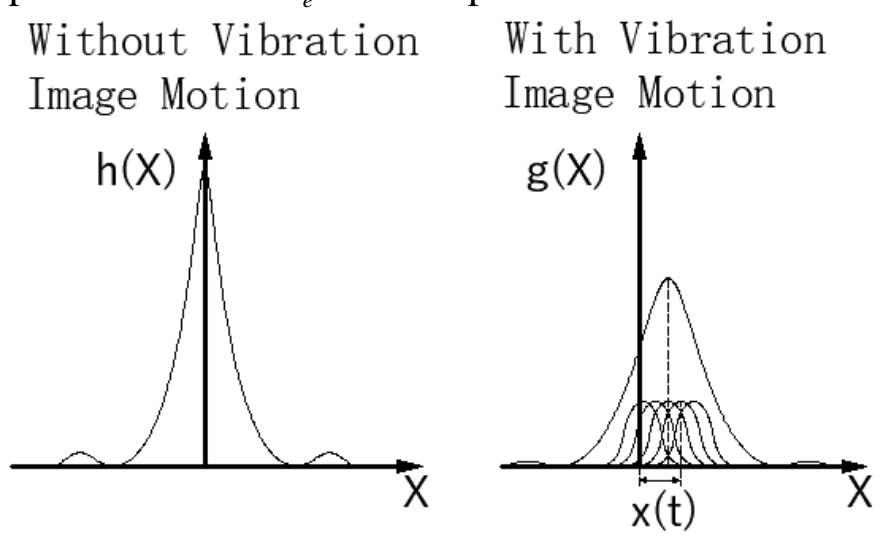

Figure 2. Diagrammatic sketch of the effect of vibration image motion on image quality

Exchange the order of integral and convolution in Equation 2,

$$
g(X)=h(X) * \frac{1}{t_{e}} \int_{t_{s}}^{t_{s}+t_{e}} \delta[X-x(t)] d t
$$

Therefore, the line spread function generated by the vibration image motion during the exposure time is:

$$
\operatorname{LSF}_{v}(X)=\frac{1}{t_{e}} \int_{t_{s}}^{t_{s}+t_{e}} \delta[X-x(t)] d t
$$

The Fourier transform of the line spread function can be used to obtain the optical transfer function under vibration conditions:

$$
O T F(f)=\int_{-\infty}^{+\infty} L S F_{v}(X) \exp (-2 j \pi f X) d X
$$

Where $\mathrm{f}$ is the spatial frequency. Substituting formula 4 into formula 5 , we can get

$$
O T F(f)=\frac{1}{t_{e}} \int_{t_{e}}^{t_{s}+t_{e}} \exp [-2 j \pi f x(t)] d t
$$


The Modulation Transfer Function(MTF) is usually used to evaluate the degree of image blur caused by vibration[11].

$$
\operatorname{MTF}(f)=\left|\frac{1}{t_{e}} \int_{t_{e}}^{t_{s}+t_{e}} \exp [-2 j \pi f x(t)] d t\right|
$$

The linear portion of the Phase Transfer Function(PTF) is used to represent the the image motion caused by the vibration.

Taylor's series expansion is performed by the integral function in formula 7 ,

$$
\exp [-2 j \pi f x(t)]=1-2 j \pi f x(t)+\frac{[-2 j \pi f x(t)]^{2}}{2 !}+\cdots+\frac{[-2 j \pi f x(t)]^{n}}{n !}+\cdots
$$

Substitute Equation 8 into Equation 7, the expression of the Optical Transfer Function is

$$
\begin{aligned}
& \operatorname{OTF}(f)=1-2 j \pi f m_{1}+\cdots+\frac{[-2 j \pi f]^{n}}{n !} m_{n}+\cdots \\
& m_{n}=\frac{1}{t_{e}} \int_{t_{s}}^{t_{s}+t_{e}} x^{n}(t) d t
\end{aligned}
$$

where $m_{n}$ is the $n^{\text {th }}$ order statistical moment of the vibration image shift function $x(t)$. By subtracting the second-order term and terms above second-order, an approximate phase transfer function can be obtained:

$$
\operatorname{PTF}(f) \approx \arctan \left(-2 j \pi f m_{1}\right)
$$

Also based on Taylor series expansion,

$$
\arctan \left(-2 j \pi f m_{1}\right)=\left(-2 j \pi f m_{1}\right)-\frac{\left(-2 j \pi f m_{1}\right)^{3}}{3 !}+\cdots+(-1) \frac{\left(-2 j \pi f m_{1}\right)^{2 n+1}}{(2 n+1) !}+\cdots
$$

By subtracting the second-order term and terms above second-order, an approximate phase transfer function can be obtained:

$$
\operatorname{PTF}(f) \approx-2 \pi f m_{1}
$$

It can be seen that the linear portion of the phase transfer function is only related to the first-order statistical moment of the image motion function during the exposure time. Aerial cameras are usually imaged using TDICCD with Pendulum sweep mechanism. The captured image is obtained by exposure of the detector at different times. Due to the difference in the overall offset of the image from the ideal position caused by the vibration at different exposure times, the image will be geometrically deformed. Since the first-order statistical moment of the image motion function during the exposure time indicates the overall offset of the image from the ideal position due to vibration, it can be used to evaluate the geometric deformation of the image.

\section{Conclusion}

The Modulation Transfer Function(MTF) is usually used to evaluate the degree of image blur caused by vibration. the linear portion of the Phase Transfer Function(PTF) is used to characterize the geometric deformation of the image. According to this model, the image quality degradation under different conditions can be analyzed more intuitively.

In the follow-up study, through the analysis of the influence of the angular vibration error of the camera and the inter-carrier damper on the target positioning accuracy, it can give measures to improve the accuracy of target positioning. 


\section{Acknowledgment}

This work was funded by International Cooperation Project of the Ministry of Science and Technology, China (No. 2016YFE0105100).

\section{References}

[1] JIA Ping, ZHANG Bao. Key Technologies and Development of Aviation Photoelectric Reconnaissance Platform[J]. Optics and Precision Engineering, 2003(01):82-88.

[2] XIU Jihong, ZHAI Linpei. Main Factors Affecting The Quality of Aerial Images[J].INFRARED(Monthly),2005(08):10-16.

[3] ZHOU Xiang-yang, LIU Wei. Vibration analysis and damping system design on an inertially stabilized platform for aerial remote sensing application [J].Journal of Chinese Inertial Technology, 2012,20(03):266-272.

[4] Sun Chongshang, Ding Yalin, Wang Dejiang, Huang Houtian, Tian Dapeng, Yao Yuan. Analysis and Verification of Influence of Sinusoidal Vibration on Imaging of Aerial Camera[J]. ACTA OPTICA SINICA, 2014,34(07):77-83.

[5] GEEG Wenbao, ZHAI Linpei, DING Yalin. .Analysis of the influence of vibration on the transfer function of optical imaging system[J].Optics and Precision Engineering,2009,17(02):314320.

[6] CHEN Linzhouting, FANG Jiancheng, GONG Xiaolin. Establishing and analysis of POS highorder error model for motion compensation in aerial remote sensing[J].Chinese Journal of Scientific Instrument,2012,33(11):2436-2445.

[7] HUANG Meng, LIU Jiayan, ZHANG Bao, MA Tianwei, Yalin. Image Motion Compensation of Small Aerial Camera System with Area Array Sensor[J].Electronics Optics\&Control,2009,16(03):68-70+74.

[8] G. D. Boreman. Modulation transfer function in the optical and electro-optical systems [M]. Bellingham, Washington, USA: SPIE press, 2001.

[9] R. D. Fiete. Modeling the Imaging Chain of Digital Cameras [M]. Bellingham, Washington, USA: SPIE press, 2001.

[10] WANG Zhile, ZHANG Xuxia, ZHANG Lanqing. Numerical calculation and analysis of dynamic MTF [J]. OPTICAL TECHNIQUE,2011,37(05): 590-596.

[11] A Friedenberg. Simulation of the MTF due to low-frequency and correlated vibrations [J]. Journal of Modern Optics, 2008, 55(3):401 